\title{
Concurrent Vital Sign and Position Sensing of Multiple Individuals Using Self-Injection-Locked Tags and Injection-Locked I/Q Receivers With Arctangent Demodulation
}

\author{
Fu-Kang Wang, Member, IEEE, Chia-Hui Fang, Tzyy-Sheng Horng, Senior Member, IEEE, \\ Kang-Chun Peng, Member, IEEE, Jian-Yu Li, Member, IEEE, and Cheng-Chung Chen
}

\begin{abstract}
This work presents a wireless system that operates in the 2.4 GHz ISM band for concurrently sensing the vital signs and positions of multiple individuals. Characterized by low complexity and high accuracy, the proposed system consists of two main parts. One is a self-injection-locked (SIL) tag carried by a subject, which emits a sinusoidal frequency-modulated (SFM) signal with vital sign information. The other is a group of injection-locked (IL) I/Q receivers, which performs arctangent demodulation of the SFM signal to obtain the position information without using RF reference signals, and simultaneously extracts the vital sign information of the subject. In the experiment, the system is capable of sensing multiple individuals based on frequency division multiple access (FDMA) technique. Moreover, fidgeting effect on the detection of vital signs has been greatly reduced by a spectral product approach. Accordingly, it is demonstrated that the experimental prototype provides accurate information about the vital signs and positions of different individuals in an indoor environment.
\end{abstract}

Index Terms-Arctangent demodulation, injection-locked (IL) I/Q receiver, self-injection-locked (SIL) tag, vital sign detection, wireless indoor positioning.

\section{INTRODUCTION}

I NDOOR positioning by wireless technologies has various applications, such as warehousing services, logistics tracking, disaster rescue, and homeland healthcare, and it has attracted increasing attention in recent years [1], [2]. Existing indoor positioning solutions, based on received signal

Manuscript received July 05, 2013; revised October 02, 2013; accepted October 16, 2013. Date of publication November 12, 2013; date of current version December 02, 2013. This work was supported in part by the National Science Council, Taiwan, under Grant 100-2221-E-110-081-MY3, Grant 100-2221-E110-082-MY3, and Grant 100-2622-E-110-001-CC1. This paper is an expanded paper from the IEEE International Microwave Symposium held on June 2-7, 2013, in Seattle, WA, USA.

F.-K. Wang, C.-H. Fang, and T.-S. Horng are with the Department of Electrical Engineering, National Sun Yat-Sen University, Kaohsiung 804, Taiwan (e-mail: d983010020@student.nsysu.edu.tw; m003010027@student.nsysu.edu.tw; jason@ee.nsysu.edu.tw).

K.-C. Peng is with Department of Computer and Communication Engineering, National Kaohsiung First University of Science and Technology, Kaohsiung 811, Taiwan (e-mail: peterpkg@ccms.nkfust.edu.tw).

J.-Y. Li, and C.-C. Chen are with the Information and Communication Research Lab., Industrial Technology Research Institute, Hsinchu 310, Taiwan (e-mail: JianYu@itri.org.tw; m400ccchen@itri.org.tw).

Color versions of one or more of the figures in this paper are available online at http://ieeexplore.ieee.org.

Digital Object Identifier 10.1109/TMTT.2013.2288224 strength (RSS), time of arrival (TOA), time difference of arrival (TDOA), round-trip time of flight (RTOF), and angle of arrival (AOA), involve mainly a mobile signal transmitter and several receiving units. The transmitted signals that are received by the various units are collected and processed to obtain information about the position of the target.

The RSS method measures the attenuation of a received signal to estimate the distance between transmitter and receiving units using a propagation equation [3]-[5]. However, its accuracy is easily affected by multipath fading and shadowing, which are caused by indoor interference [3]. In a TOA system, all of the receiving units and transmitter are synchronized in time [6]-[8]. One-way propagation delay is measured to calculate the distance between the transmitter and each receiving unit. Therefore, the target can be located from the intersection of the circles that are centered at receiving units. Unlike the TOA method, the TDOA method [9]-[11] measures the difference between the arrival times at multiple receiving units to locate a target. The benefit is that time synchronization is required only among the receiving units.

Although the TOA and TDOA methods have been widely used in indoor positioning systems because of their high accuracy, the error in reference signal synchronization significantly degrade their performance [12]. However, designing a synchronization mechanism for simultaneously achieving accuracy, energy efficiency, system complexity, scalability, and robustness remains challenging [6].

The time synchronization requirements are not necessary in the RTOF and AOA methods. In an RTOF system, the receiving units are replaced with ranging radars, such as pulsed radars [13] or frequency-modulated continuous wave (FMCW) radars [14]. Moreover, the target carries a transponder that often utilizes passive frequency doubler [13]-[16] to respond to the interrogating radar signal, and the round-trip propagation delay is measured to determine the position of the target. However, the propagation loss leads to a trade-off between sensing range and radar transmitting power. Recently, in [17] and [18], a switched injectionlocked oscillator is used as an active backscatter transponder in a localization system to significantly amplify the response signal and hence increase the sensing range. Nonetheless, a large receiving signal bandwidth is required in this system, which is detrimental to receiver sensitivity. Whereas the aforementioned 
methods involve at least three receiving units for two-dimensional positioning, the AOA system [19], [20] requires only two receiving units with a high-directivity phased array antenna to locate the desired target from the intersection of two antenna beam directions, and no synchronization mechanism is required. However, the use of phased array antennas to ensure high accuracy is associated with the drawbacks of increased system complexity and excessive cost.

CW radars have been extensively used for accurate and reliable non-contact cardiopulmonary monitoring [21]. With the help of FMCW and stepped frequency CW (SFCW) technologies [22], [23], these radars can concurrently localize the subjects while monitoring their motion and health. Recently, the use of self-injection locked (SIL) radars for sensing vital signs has been developed as a method with favorable performance, low system complexity, high sensitivity, and high immunity against the clutter effect [24], [25]. Additionally, a hopping frequency can be used to solve the problems of null points and co-frequency interference. Moreover, the effects of random body motion can be eliminated by the mutual injection locking of two SIL radars [25]. Advanced see-through-wall surveillance system can also be built by an SIL radar with FMCW and tracking antenna technologies [26].

Our preliminary work [27] as the first to combine TDOA and SIL radar technologies to sense simultaneously the vital signs and position of a subject. The system comprises an SIL tag and three receivers. Each receiver includes an amplifier limiter, an injection-locked oscillator (ILO), and a delay discriminator to demodulate a sinusoidal frequency-modulated (SFM) signal that is emitted from an SIL tag. Since there are no RF reference signals applied to the receivers, complex reference signal synchronization issues are avoided. However, delay imbalance among various receivers significantly degrades positioning accuracy. Therefore, amplifier limiters are utilized in [27] to maintain a similar injection power level and effective time delay of the ILO in each receiver.

In this paper, injection-locked (IL) I/Q architecture with arctangent demodulation is adopted. Basically, the output TDOA information is not influenced by the delays of ILOs, so the amplifier limiters are unnecessary, and the system complexity and robustness are improved. Moreover, this work adopts frequency division multiple access (FDMA) technique to sense multiple individuals. As shown in Fig. 1, the tags attached to various subjects are operated at different frequencies, and the operating frequencies of all receivers are easily adjusted to extract vital signs and positions of multiple subjects.

\section{System ARChitecture ANd PRINCIPLE of Detection}

Figs. 2(a) and 2(b) respectively present block diagrams of the SIL tag and the IL I/Q receiver of the proposed sensing system for concurrent vital sign detection and indoor positioning. The SIL tag is constructed from a tag antenna and an injectionlocked oscillator (ILO) that uses a common terminal for injection and output signals. This antenna is designed as a planar inverted-F antenna with a gain of $0 \mathrm{dBi}$ in the $2.4 \mathrm{GHz}$ ISM band. The tuning range of the ILO in the tag is from 2.4 to $2.484 \mathrm{GHz}$, and the tag delivers an output power of approximately $5 \mathrm{dBm}$.

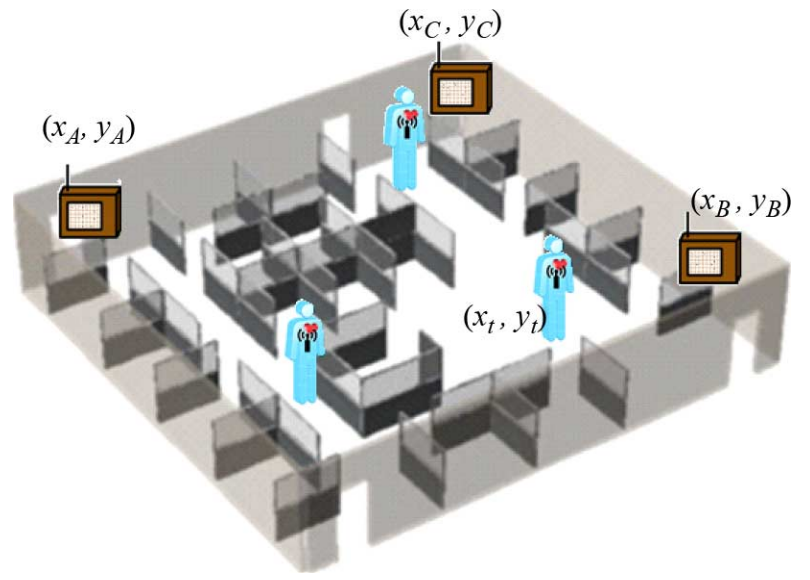

Fig. 1. Proposed system for concurrent vital sign and indoor position sensing.

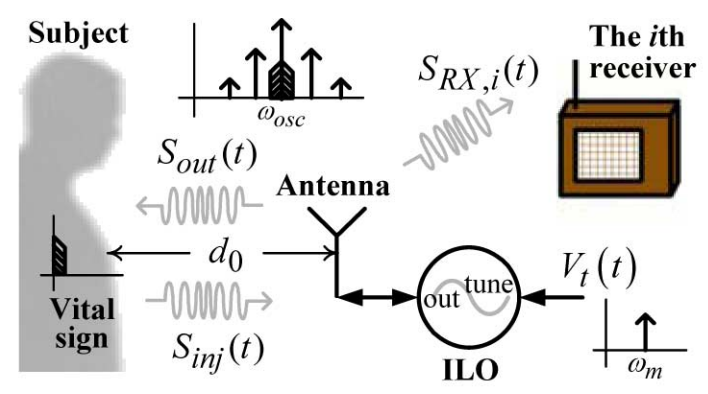

(a)

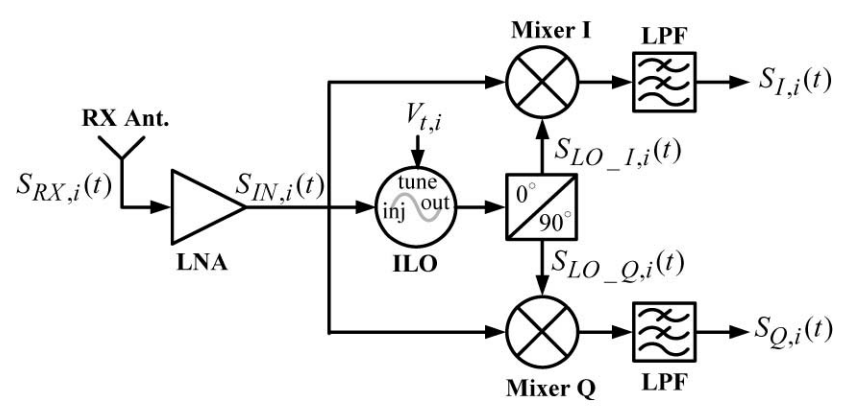

(b)

Fig. 2. Block diagrams of the proposed system. (a) SIL tag. (b) IL I/Q receiver.

The IL I/Q receiver mainly contains a receiving (RX) antenna, a low-noise amplifier (LNA) with a power gain of $22 \mathrm{~dB}$, an ILO with a dedicated injection input terminal, a quadrature power splitter, I/Q mixers, and two low-pass filters (LPFs). The RX antenna is a panel antenna with a high gain of $12 \mathrm{dBi}$. The ILO has a tuning range from 2.3 to $2.6 \mathrm{GHz}$ and it delivers an output power of about $5 \mathrm{dBm}$ to pump the I/Q mixers with a conversion gain of $-6.6 \mathrm{~dB}$. The principle of detection is described briefly as follows.

As shown in Fig. 1, three IL I/Q receivers with known locations in the sensing range determine the positions of the target subjects who are carrying SIL tags. Notably, the tags are tied to the chest of the subjects with the help of the belts. First, with reference to Fig. 2(a), the tuning input voltage $V_{t}(t)$, a sinusoidal signal, frequency-modulates the inherent oscillation signal $S_{\text {osc }}(t)$ of the ILO for two purposes. One is for frequency separation of the individual tags and the other is for 
generation of the modulated localization signal. The tag antenna can transmit the ILO output signal $S_{\text {out }}(t)$ to the environment and receive injection signal $S_{i n j}(t)$ that is reflected back from the subject with a Doppler modulation due to the subject's cardiopulmonary motion. Next, the injection signal enters the ILO via its output terminal to establish an SIL loop.

Second, with reference to Fig. 2(b), the SFM signals emitted from the SIL tags are received by RX antenna of the $i$ th IL I/Q receiver as $S_{R X, i}(t)$; the low-noise amplified signal $S_{I N, i}(t)$ is fed into the RF terminals of the I/Q mixers and the injection terminal of the ILO that enters an IL state after receiving $S_{I N, i}(t)$. In the receiver, the ILO functions as a band-pass filter with tunable time delay. Then, the quadrature power splitter outputs $S_{L O_{-}, i}(t)$ and $S_{L O_{-} Q, i}(t)$ to the LO ports of the I/Q mixers. Essentially, this receiver is a non-coherent frequency demodulator that outputs low-pass filtered signals $S_{I, i}(t)$ and $S_{Q, i}(t)$ related to the instantaneous frequency variation of the received signal $S_{R X, i}(t)$.

Third, with the help of digital signal processing (DSP), the baseband signal of the $i$ th receiver $S_{b b, i}(t)$ is obtained via an arctangent function. Finally, vital signs, including respiration and heart rates, are extracted from the low-pass filtered baseband signals, whereas the position of the subject is computed from the differences between the phases of the high-pass filtered baseband signals in different receivers.

\section{A. SIL Tag}

As shown in Fig. 2(a), the SIL tag has a single-antenna architecture: it uses the same antenna to transmit $S_{\text {out }}(t)$ as it does to receive $S_{i n j}(t)$ without the need to isolate both signals. The transmit-receive coupling hardly influences the signal-to-noise ratio of the SIL tag [25]. The ILO in a tag has an inherent instantaneous oscillation frequency, $\omega_{o s c}(t)$, and a constant oscillation amplitude, $E_{o s c}$. When an injection signal $S_{i n i}(t)$, with instantaneous frequency $\omega_{i n j}(t)$ and constant amplitude $E_{i n j}$, enters the ILO, the output signal $S_{i n j}(t)$, with constant amplitude $E_{\text {out }}$, can be regarded as a vector that rotates clockwise with beat frequency $d \alpha(t) / d t$ with respect to $S_{i n j}(t)$, while $\alpha(t)$ is the phase difference from $S_{i n j}(t)$ to $S_{\text {osc }}(t)$. Based on Adler's analysis [28], the instantaneous output frequency $\omega_{\text {out }}(t)$ is represented as

$$
\omega_{\text {out }}(t)=\omega_{\text {osc }}(t)-\omega_{L R} \sin \alpha(t) .
$$

When a sinusoidal signal $V_{t} \sin \omega_{m} t$ is applied to the tuning terminal, the inherent oscillation frequency becomes

$$
\omega_{o s c}(t)=\omega_{c}+2 \pi K_{v} V_{t} \sin \omega_{m} t
$$

where $K_{v}$ is the tuning sensitivity and $\omega_{c}$ is the center frequency of the ILO. Based on the assumption that the ILO has a small tuning range, the locking range $\omega_{L R}$ in (1) is given by

$$
\omega_{L R}=\frac{\omega_{c}}{2 Q_{\mathrm{tank}}} \frac{E_{i n j}}{E_{o s c}}
$$

where $Q_{\text {tank }}$ represents the quality factor of ILO's tank circuit.
In an SIL state of sensing cardiopulmonary activity, the phase difference $\alpha(t)$ equals the round-trip phase delay between the tag and the subject's chest, given by

$$
\alpha(t)=\frac{2 \omega_{c}}{c}\left(d_{0}+x_{b}(t)\right) .
$$

In (4), $d_{0}$ is the initial distance from the subject to the tag; $x_{b}(t)$ represents the displacement fluctuation that is caused by the cardiopulmonary motion of the subject, and $c$ denotes the speed of light.

The frequency variation that is caused by the SIL phenomenon, included in (1), has been demonstrated in [24]-[26]. The experimental results in these previous works indicate the satisfactory sensing of cardiopulmonary movements by SIL radar. Obviously, the proposed tag is very low complexity, and therefore is likely to consume much less power than the conventional tags using contact sensors with wireless data transmission capabilities [29].

\section{B. IL I/Q Receiver}

As shown in Fig. 2(b), the $S_{R X, i}(t)$ that is received in the $i$ th receiver is amplified as the input signal $S_{I N, i}(t)$, which can be expressed as

$$
\begin{aligned}
S_{I N, i}(t)=E_{I N, i} & \cos \left(\omega_{c}\left(t-\tau_{i}\right)-\omega_{L R} \int_{0}^{t-\tau_{i}} \sin \alpha\left(t^{\prime}\right) d t^{\prime}\right. \\
& \left.+2 \pi K_{v} V_{t} \int_{0}^{t-\tau_{i}} \sin \omega_{m} t^{\prime} d t^{\prime}+\theta_{0, i}\right)
\end{aligned}
$$

where $E_{I N, i}$ and $\theta_{0, i}$ are the constant amplitude and initial phase of the input signal, respectively. In (5), $\tau_{i}$ represents the propagation delay, which is given by $d_{p, i} / c$, where $d_{p, i}$ is the distance between the $i$ th receiver and the tag.

The input signal is simultaneously fed into the input terminals of the I/Q mixers and the ILO in the $i$ th receiver. When the ILO is injection-locked by $S_{I N, i}(t)$, it functions as a band-pass filter with tunable time delay, and its output signal is quadrature-divided as $S_{L O_{-}, i}(t)$ and $S_{L O_{-} Q, i}(t)$ to pump the I/Q mixers. Basically, the signals $S_{L O_{-} I, i}(t)$ and $S_{L O \_O, i}(t)$ are just the delayed and scaled versions of (5) with or without 90 degree phase shift. In channel I, the local signal $S_{L_{-} I, i}(t)$ is given by

$$
\begin{aligned}
& S_{L O \_, i}(t)=\frac{1}{\sqrt{2}} E_{I L O, i} \cos \left(\omega_{c}\left(t-\tau_{i}-\tau_{I L, i}\right)\right. \\
& -\omega_{L R} \int_{0}^{t-\tau_{i}-\tau_{I L, i}} \sin \alpha\left(t^{\prime}\right) d t^{\prime} \\
& \left.+2 \pi K_{v} V_{t} \int_{0}^{t-\tau_{i}-\tau_{I L, i}} \sin \omega_{m} t^{\prime} d t^{\prime}+\theta_{0, i}\right)
\end{aligned}
$$

where $E_{I L O, i}$ and $\tau_{I L, i}$ are the output voltage and lock-in time, respectively, of the ILO in the $i$ th receiver. According to [30], $\tau_{I L, i}$ is given by

$$
\tau_{I L, i}=\left(\omega_{L R, i}^{2}-\Delta \omega_{o s c}^{2}\right)^{-0.5}
$$

where $\omega_{L R, i}$ is the locking range of the ILO in the $i$ th receiver. $\Delta \omega_{o s c}$ is the initial frequency difference between the tag and the $i$ th receiver. 
Next, mixer I outputs the product of $S_{L O \_I, i}(t)$ and $S_{I N, i}(t)$. After low-pass filtering of spurious signals, the output signal of channel I in the $i$ th receiver $S_{I, i}(t)$ can be found as

$$
\begin{aligned}
S_{I, i}(t)=G_{c, i} E_{I N, i} & \cos \left(\omega_{c} \tau_{I L, i}-\omega_{L R} \int_{t-\tau_{i}-\tau_{I L, i}}^{t-\tau_{i}} \sin \alpha\left(t^{\prime}\right) d t^{\prime}\right. \\
& \left.+2 \pi K_{v} V_{t} \int_{t-\tau_{i}-\tau_{I L, i}}^{t-\tau_{i}} \sin \omega_{m} t^{\prime} d t^{\prime}\right)
\end{aligned}
$$

where $G_{c, i}$ represents the conversion gain of I/Q mixers.

Assume that $\omega_{m} \tau_{I L, i} \ll 2 \pi, S_{I, i}(t)$ can be approximated as

$$
\begin{aligned}
S_{I, i}(t) \approx G_{c, i} E_{I N, i} & \cos \left(\omega_{c} \tau_{I L, i}-\omega_{L R} \tau_{I L, i} \sin \alpha\left(t-\tau_{i}\right)\right. \\
+ & \left.2 \pi K_{v} V_{t} \tau_{I L, i} \sin \omega_{m}\left(t-\tau_{i}\right)\right) .
\end{aligned}
$$

In channel Q, the local signal $S_{L O_{-} Q, i}(t)$ is an orthonormal version of $S_{L O \_I, i}(t)$ :

$$
\begin{aligned}
S_{L O \_Q, i}(t)= & e^{i \pi / 2} \cdot S_{L O \_I, i}(t) \\
= & \frac{-1}{\sqrt{2}} E_{I L O, i} \sin \left(\omega_{c}\left(t-\tau_{i}-\tau_{I L, i}\right)\right. \\
& -\omega_{L R} \int_{0}^{t-\tau_{i}-\tau_{I L, i}} \sin \alpha\left(t^{\prime}\right) d t^{\prime} \\
& \left.+2 \pi K_{v} V_{t} \int_{0}^{t-\tau_{i}-\tau_{I L, i}} \sin \omega_{m} t^{\prime} d t^{\prime}+\theta_{0, i}\right) .
\end{aligned}
$$

Similarly, the output signal of channel Q in the $i$ th receiver $S_{Q, i}(t)$ is approximated by

$$
\begin{aligned}
S_{Q, i}(t) \approx-G_{c, i} E_{I N, i} & \sin \left(\omega_{c} \tau_{I L, i}-\omega_{L R} \tau_{I L, i} \sin \alpha\left(t-\tau_{i}\right)\right. \\
+ & \left.2 \pi K_{v} V_{t} \tau_{I L, i} \sin \omega_{m}\left(t-\tau_{i}\right)\right) . \quad(11)
\end{aligned}
$$

Notably, small angle approximation is not valid to use in (9) and (11) because the term $\omega_{c} \tau_{I L, i}$ may not approach 0 or $\pi / 2$. Therefore, the output signals fail to reflect the change in frequency that is caused by the tuning voltage $V_{t}(t)$ and the cardiopulmonary motion of the subject. Moreover, according to (7), the lock-in time of the ILO $\tau_{I L, i}$ is correlated with the locking range $\omega_{L R, i}$ that is dependent on the input voltage to the ILO, and the distances between the tag and the various receivers are typically different, yielding different values of $E_{I N, i}$ and $\tau_{I L, i}$ for different $i$. This ILO delay mismatch affects the differences between the phases of the baseband signals that are delivered by the different receivers, causing a degradation of accuracy in localization. In this study, the arctangent demodulation method proposed in [31] is used to solve the above problem. The baseband signal $S_{b b, i}(t)$ is defined and calculated according to (9) and (11), as shown by

$$
\begin{aligned}
S_{b b, i}(t)= & \arctan \left(\frac{-S_{Q, i}(t)}{S_{I, i}(t)}\right) \\
\approx \pi & \cdot \operatorname{Mod}\left(\omega_{c} \tau_{I L, i}, \pi\right) \\
& -\omega_{L R} \tau_{I L, i} \sin \alpha\left(t-\tau_{i}\right) \\
& +2 \pi K_{v} V_{t} \tau_{I L, i} \sin \omega_{m}\left(t-\tau_{i}\right)
\end{aligned}
$$

where Mod denotes a modulus function. Clearly, $\tau_{I L, i}$ does not affect the phase of ac components in the baseband signal. Thus, the delay imbalance due to different $\tau_{I L, i}$ values for different $i$ does not alter the phase difference between the baseband signals, and the arctangent demodulation scheme can improve the robustness of the positioning system.

\section{Principles of Vital Sign and Position Detection}

The sensing process is described as follows. First, the baseband signal is split by low-pass and high-pass filtering. That is, the baseband signal can be rewritten as

$$
S_{b b, i}(t)=S_{L P F, i}(t)+S_{H P F, i}(t)
$$

where $S_{L P F, i}(t)$ and $S_{H P F, i}(t)$ represent the low-pass filtered and high-pass filtered baseband signal. Substituting (4) into (12), and assuming that $2 \omega_{o s c} x_{b}(t) / c \ll 1$, enables $S_{L P F_{i}}(t)$ to be approximated by

$$
\begin{aligned}
S_{L P F, i}(t) \approx & \cdot \operatorname{Mod}\left(\omega_{c} \tau_{I L, i}, \pi\right) \\
& -\omega_{L R} \tau_{I L, i} \sin \left(\frac{2 \omega_{c} d_{0}}{c}\right) \\
& -\frac{2 \omega_{c} \omega_{L R} \tau_{I L, i}}{c} \cos \left(\frac{2 \omega_{c} d_{0}}{c}\right) x_{b}\left(t-\tau_{i}\right)
\end{aligned}
$$

which includes the dc level and the Doppler signal that is caused by cardiopulmonary motion. To eliminate the dc level, the term $\omega_{c} \tau_{I L, i}$ must equal an integer multiple of $\pi$ by adjusting $\tau_{I L, i}$ via the ILO tuning voltage $V_{t, i}$. Moreover, $d_{0}$ should be kept as minimum as possible by fastening the tag belt very tightly around the chest. Since the propagation delay is generally less than the period of the vital sign of interest, $x_{b}\left(t-\tau_{i}\right)$ can be approximated as $x_{b}(t)$, and the low-pass filtered baseband signals from which the vital sign information is extracted are almost identical at all three receivers.

The high-pass filtered baseband signal $S_{H P F, i}(t)$ in (13) is given by

$$
S_{H P F, i}(t)=2 \pi K_{v} V_{t} \tau_{I L, i} \sin \omega_{m}\left(t-\tau_{i}\right) .
$$

The sine function described by (15) represents a delayed version of the sinusoidal modulation signal that is applied to the ILO of the tag. Moreover, the TDOA between the $i$ th and $j$ th receiver is proportional to the phase difference between $S_{H P F, i}(t)$ and $S_{H P F, j}(t)$, which is described by

$$
\begin{aligned}
\Delta \phi_{i, j} & =\phi_{i}-\phi_{j}=-\omega_{m}\left(\tau_{i}-\tau_{j}\right) \\
& =-\frac{\omega_{m}}{c}\left(d_{p, i}-d_{p, j}\right)
\end{aligned}
$$

where $d_{p, i}$ and $d_{p, j}$ are the distance from the tag to the $i$ th and $j$ th receiver, respectively.

Fig. 3 presents the positioning principle. For two-dimensional positioning, the experimental environment includes three receivers (RX_A, RX_B, and RX_C). The coordinates of RX_A are $\left(x_{A}, y_{A}\right)$; those of RX_B are $\left(x_{B}, y_{B}\right)$, and those of RX_C are $\left(x_{C}, y_{C}\right)$. After arctangent demodulation of the SFM signals 


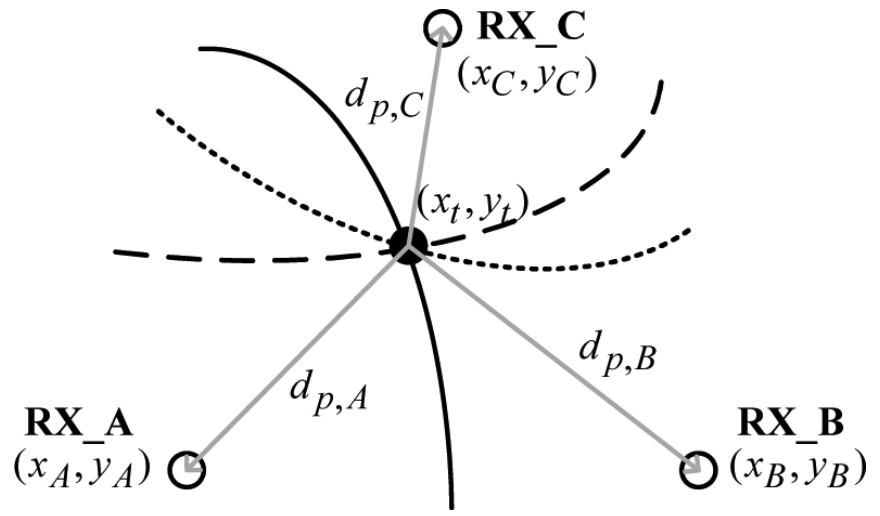

Fig. 3. Positioning principle using TDOA method.

that are emitted from the tag with unknown position, hyperbolas are plotted using the following equations:

$$
\begin{aligned}
& \sqrt{\left(x-x_{A}\right)^{2}+\left(y-y_{A}\right)^{2}}-\sqrt{\left(x-x_{B}\right)^{2}+\left(y-y_{B}\right)^{2}} \\
& =-\frac{c \Delta \phi_{A, B}}{\omega_{m}} \\
& \sqrt{\left(x-x_{B}\right)^{2}+\left(y-y_{B}\right)^{2}}-\sqrt{\left(x-x_{C}\right)^{2}+\left(y-y_{C}\right)^{2}} \\
& =-\frac{c \Delta \phi_{B, C}}{\omega_{m}} \\
& \sqrt{\left(x-x_{C}\right)^{2}+\left(y-y_{C}\right)^{2}}-\sqrt{\left(x-x_{A}\right)^{2}+\left(y-y_{A}\right)^{2}} \\
& =-\frac{c \Delta \phi_{C, A}}{\omega_{m}} .
\end{aligned}
$$

The point of their intersection $\left(x_{t}, y_{t}\right)$ is the location of the detected tag.

\section{EXPERIMENTAL SETUP AND VALIDATIONS}

Fig. 4(a) presents a photograph of the SIL tag that was used in the experiment. The ILO adopts a single-ended Colpitts architecture and consumes a dc power of $20 \mathrm{~mW}$. The $5-\mathrm{dBm}$ output power of the ILO is fed into the planar inverted $\mathrm{F}$ antenna. The tag occupies an area of $4.8 \times 2.4 \mathrm{~cm}^{2}$. A $2.5-\mathrm{MHz}$ sinusoidal signal enters the tuning terminal of the ILO in the tag to generate an SFM signal with a modulation bandwidth of $7 \mathrm{MHz}$. Notably, a higher modulation frequency yields a larger phase difference between the output baseband signals at the receivers. However, limited by the tuning speed of the ILO, the modulation frequency of $2.5 \mathrm{MHz}$ is the highest that can be set for the SFM signal under a satisfactory signal-to-noise ratio.

Fig. 4(b) shows a photograph of the IL I/Q receiver, which is assembled by several coaxial components, including an LNA, two power splitters, a pair of I and Q mixers, a 90-degree hybrid, two LPFs, and an ILO. The ILO used in this receiver is basically the same as that for the tag with an output power of about $5 \mathrm{dBm}$. It separates an injection and LO signal path to the ILO output port via a circulator.

The operation of the IL I/Q receiver is verified by obtaining the consistent arctangent-demodulation results between theory and measurement. The experimental setup connects the LNA input directly to the Agilent E4433B ESG digital RF signal

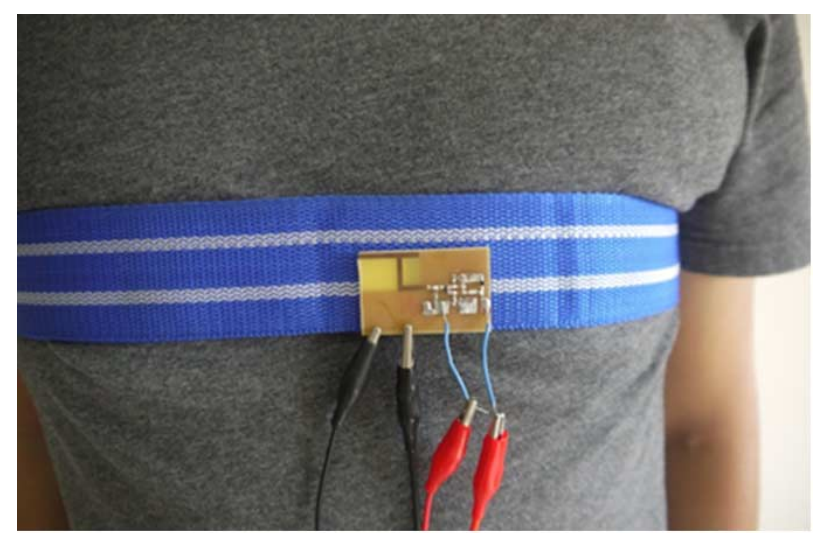

(a)

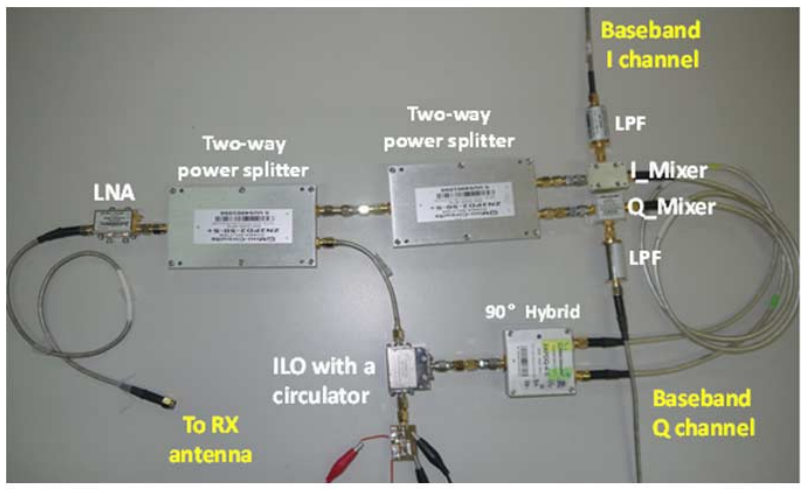

(b)

Fig. 4. Photographs of the system hardware. (a) The SIL tag tied to the chest of a subject. (b) The IL I/Q receiver assembled by coaxial components.

generator. In the experiment, the generator outputs an SFM signal with a carrier frequency of $2.41 \mathrm{GHz}$ and an output power of $-40 \mathrm{dBm}$. The modulation rate $\omega_{m} / 2 \pi$ and the frequency deviation $K_{v} V_{t}$ are 2.5 and $1 \mathrm{MHz}$, respectively. The propagation delay $\tau_{i}$ is zero, and the frequency variation of $\omega_{L R} \sin \alpha\left(t-\tau_{i}\right)$, caused by subject's cardiopulmonary activity, is not considered in this experiment. The ILO with a tank quality factor of approximately 5.2 adjusts its inherent oscillation frequency of the IL I/Q receiver to $2.409 \mathrm{GHz}$, such that the locking range and the initial frequency difference are 16 and $1 \mathrm{MHz}$, respectively. Hence the ILO can provide a time delay $\left(\tau_{I L, i}\right)$ of $9.96 \mathrm{~ns}$ based on (7), and the passband frequency of the LPFs is dc to $23 \mathrm{MHz}$. With this specific value of $\tau_{I L, i}, \omega_{c} \tau_{I L, i}$ in this experiment is an integer multiple of $\pi$. Figs. 3(a) and 3(b) plot the measured output signals in channels I and Q, respectively. As revealed in (9) and (11), the I-channel signal $S_{I}(t)$ shown in Fig. 5(a) exhibits a significant dc component of $1.197 \mathrm{~V}$ and a peak-to-peak amplitude of $0.031 \mathrm{~V}$. Moreover, the waveform fluctuates at double the frequency of modulation rate. In contrast, the Q-channel signal $S_{Q}(t)$ plotted in Fig. 5(b) varies at the frequency of modulation rate with a de value of $0.004 \mathrm{~V}$ and a peak-to-peak amplitude of $0.536 \mathrm{~V}$. Therefore, $S_{I}(t)$ is distorted quite obviously while $S_{Q}(t)$ is distorted only slightly. This phenomenon is similar to the so-called null and optimal points of a single-channel 


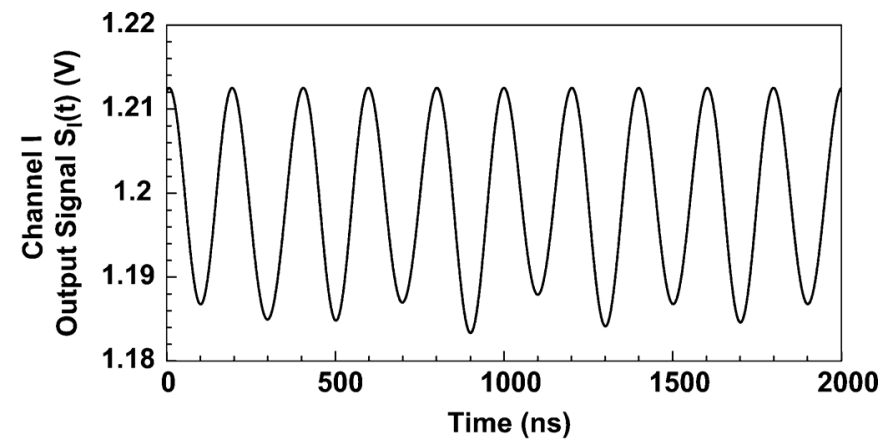

(a)

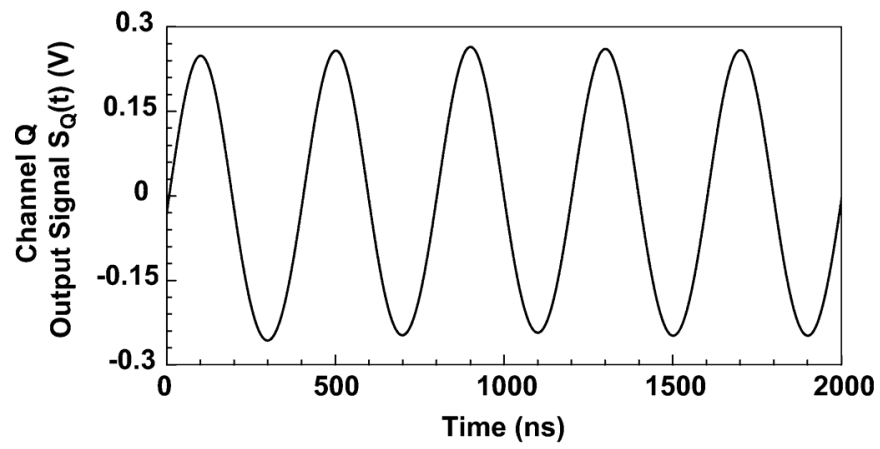

(b)

Fig. 5. Output signals of the IL I/Q receiver. (a) Channel I. (b) Channel Q.

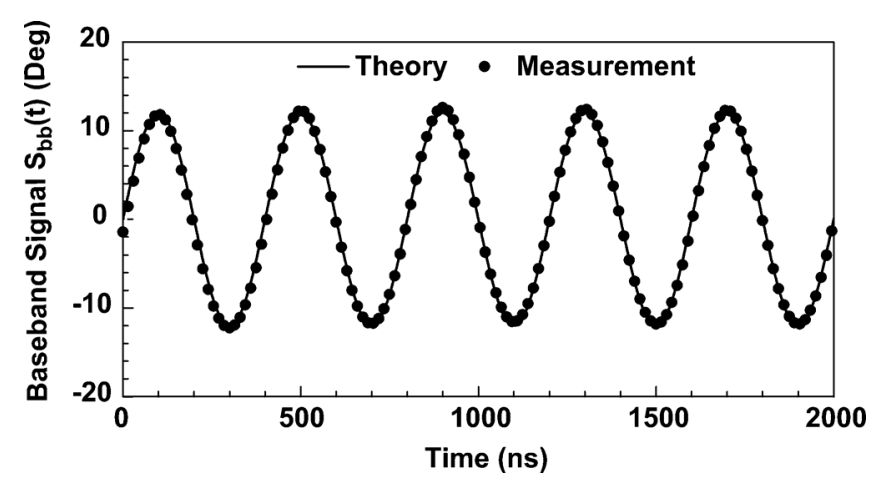

Fig. 6. Output signal of the IL I/Q receiver using arctangent demodulation.

Doppler radar [31]. In short, the single channel receiver is limited by the fact that the phase term $\omega_{c} \tau_{I L, i}$ greatly influences the waveform of the output signal.

Fig. 6 plots the baseband signals that are obtained by arctangent demodulation using DSP technique. It should be cautioned that the dc offset generated by self-mixing of the ILO leakage must be calibrated out before performing arctangent demodulation in the DSP program [31]. In Fig. 6, the solid line plots the phase change that is calculated from (12): the dc component and the amplitude of the ac component are 0 and 12 degrees, respectively. In Fig. 6, the measurements are represented as circles, showing excellent agreement with the calculated predictions. Importantly, simultaneously considering both of the output signals in the I/Q channels with arctangent demodulation reveals that the baseband signal can precisely reflect the instantaneous frequency variation of the input SFM signal, regardless of the delay of ILO.

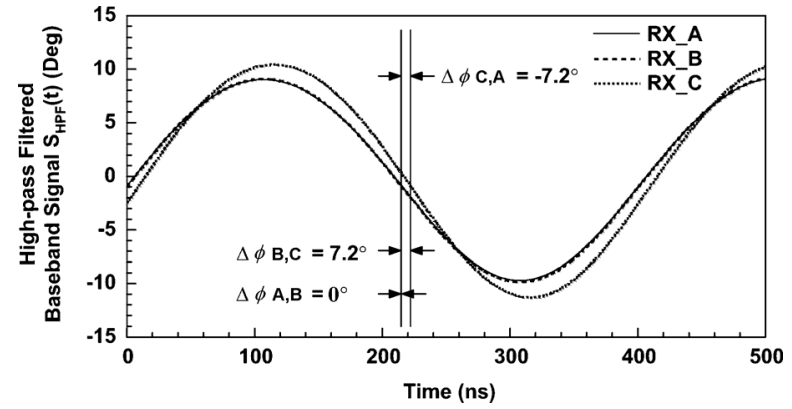

Fig. 7. High-pass filtered baseband signals for positioning subject 1.

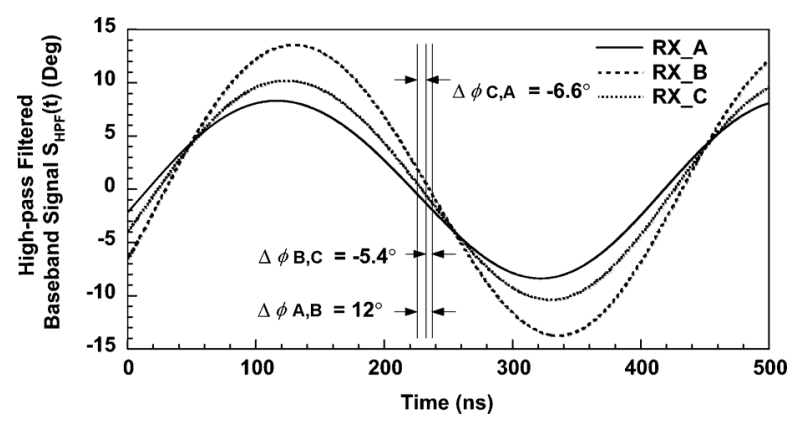

Fig. 8. High-pass filtered baseband signals for positioning subject 2 .

\section{SEnSing Results AND Discussion}

The sensing environment is an $8 \times 8 \mathrm{~m}^{2}$ cubicle office that contains various objects. The three subjects carry tags, breathe normally, and stand at coordinates $(4,0.5),(1.5,2.5)$, and $(4.8$, 4.5) (m), respectively. For an SIL tag, this work simultaneously senses multiple individuals by using FDMA technique to avoid the clock synchronization protocol and mutual injection pulling issues present in competing techniques such as time division multiple access (TDMA) and code division multiple access (CDMA). The tags are operated at $2.41 \mathrm{GHz}$ for subject $1,2.44 \mathrm{GHz}$ for subject 2 , and $2.47 \mathrm{GHz}$ for subject 3 . The receivers, RX_A, RX_B, and RX_C, are located at $(0,0),(8,0)$, and $(4,4 \sqrt{3})(\mathrm{m})$, respectively. To detect the positions and vital signs of multiple individuals, the IL I/Q receivers adjust the initial frequencies of the ILOs to $2.409 \mathrm{GHz}$ for subject $1,2.439$ $\mathrm{GHz}$ for subject 2, and $2.469 \mathrm{GHz}$ for subject 3 to demodulate the SFM signals from the different tags. Accordingly, $\Delta \omega_{o s c}$ in (7) is $2 \pi \times 1 \mathrm{MHz}$.

To isolate vital sign and position information from the baseband signal, the cut-off frequencies for low-pass and high-pass filtering are set to $10 \mathrm{~Hz}$ and $2 \mathrm{MHz}$, respectively. Figs. 7-9 plot the high-pass filtered baseband signals for the indoor positioning of multiple individuals. The solid line, broken line, and dotted line represent the signal from RX_A, RX_B, and RX_C, respectively.

For subject 1, the distances between the tag and the receivers, $d_{p 1, A}, d_{p 1, B}$, and $d_{p 1, C}$, are $4.03,4.03$, and $6.43 \mathrm{~m}$, respectively. Since $d_{p 1, A}$ equals $d_{p 1, B}$, the waveforms of $S_{H P F, A}(t)$ and $S_{H P F, B}(t)$ in Fig. 7 coincide. The phase differences, $\Delta \phi_{A, B}$, $\Delta \phi_{B, C}$, and $\Delta \phi_{C, A}$ are calculated to be $0^{\circ}, 7.2^{\circ}$, and $-7.2^{\circ}$, respectively. From (17)-(19), the point of intersection yields the location of the subject as $(4,0.497)(\mathrm{m})$; As shown in Fig. 8, 


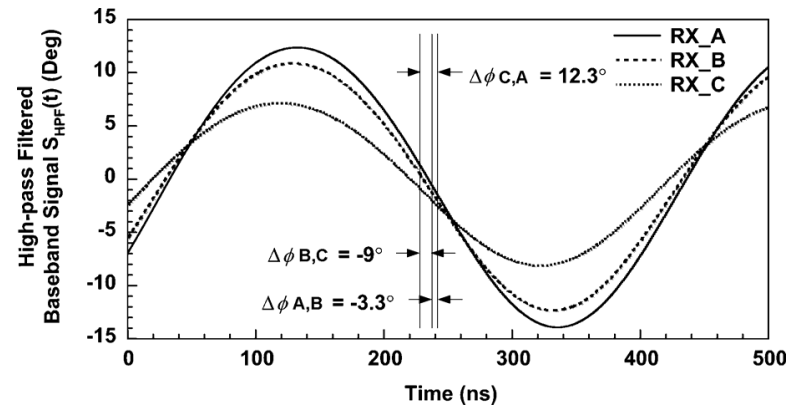

Fig. 9. High-pass filtered baseband signals for positioning subject 3.

TABLE I

COMPARISONS OF POSITIONING RESUltS FOR DifFERENT SUBJECTS.

\begin{tabular}{cccc}
\hline \hline & Subject 1 & Subject 2 & Subject 3 \\
\hline $\begin{array}{c}\text { Actual } \\
\text { position (m) }\end{array}$ & $(4,0.5)$ & $(1.5,2.5)$ & $(4.8,4.5)$ \\
$\begin{array}{c}\text { Estimated } \\
\text { position (m) }\end{array}$ & $(4,0.497)$ & $(1.549,2.454)$ & $(4.836,4.538)$ \\
\hline Error (cm) & 0.3 & 6.7 & 5.2 \\
\hline \hline
\end{tabular}

$\Delta \phi_{A, B}, \Delta \phi_{B, C}$, and $\Delta \phi_{C, A}$ are $12^{\circ},-5.4^{\circ}$, and $-6.6^{\circ}$, respectively, and the position of subject 2 is therefore calculated to be $(1.549,2.454)(\mathrm{m})$; Similarly, the calculated position of subject 3 is $(4.836,4.538)(\mathrm{m})$ since $\Delta \phi_{A, B}, \Delta \phi_{B, C}$, and $\Delta \phi_{C, A}$ in Fig. 9 are $-3.3^{\circ},-9^{\circ}$, and $12.3^{\circ}$, respectively. Table I presents the actual and estimated positions of the subjects. The errors, $0.3,6.7$, and $5.2 \mathrm{~cm}$, reveal good agreements. In this study, more than 10 measurements were taken by varying subject positions. The distance error in the worst case is $10 \mathrm{~cm}$. The sensing range can be further expanded by increasing the output power of the tag and the LNA gain of the receivers, or installing more receivers.

In the proposed system, a simple time-division multiplexing scheme is used to sense multiple individuals by switching all the channels in turn in order to collect the signal samples from different channels easily. In the actual measurement, the channel switching and sampling time is set at 0.01 and $0.1 \mathrm{~s}$, respectively. Accordingly, the system allows a maximum capacity of 10 people.

Indoor environments affect the positioning accuracy in a localization system more than do outdoor ones because of multipath and non-line-of-sight (NLOS) effects. It can be straightforward to expand the proposed receiver architecture with spatial diversity to improve the multipath fading. The other challenge is the NLOS problem that is based on the assumption that the path between the tag and part of the receivers is obscured. To solve this problem, first, the sensitivity and density of the receivers must be increased, and then the LOS and NLOS signals are collected and processed, using existing algorithms [32] to find the actual position of the detected target.

Fig. 10 plots the signal waveform of the sensed vital signs of subject 1 . The irregular fluctuation in the period between 27 and $30 \mathrm{~s}$ is caused by the subject's fidgeting movements. The solid line, broken line, and dotted line in Fig. 11(a) represent the spectra in Fig. 10 with different 10-s periods. Clearly, the heartbeat signals are not always distinguishable from random body

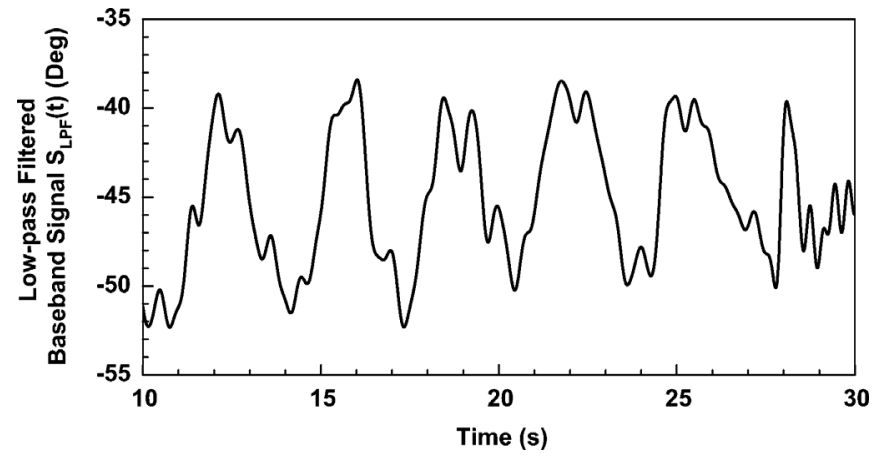

Fig. 10. Low-pass filtered baseband signal for extracting subject 1's vital sign information.

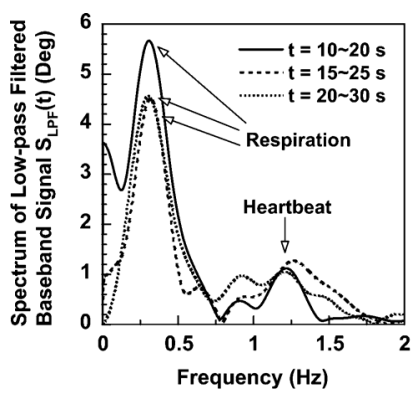

(a)

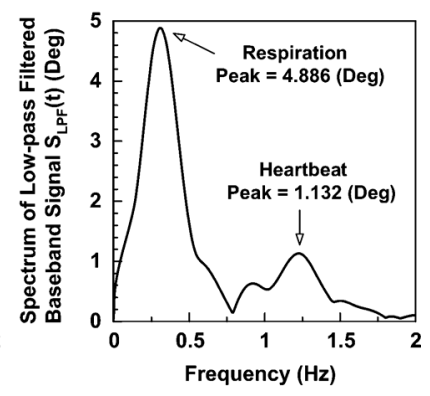

(b)
Fig. 11. Spectra of low-pass filtered baseband signal (a) Original spectra from the Fourier transform of the waveform of Fig. 10 at different 10-s intervals (b) Improved spectrum using (20).

motion signals. Therefore, this work improves the canceling of random body motion based on autocorrelation concept by using a spectral product approach, which is expressed as

$$
\begin{aligned}
S_{L P F, i}(\omega)= & \left\{\mathbb{F}\left(\left[\Pi\left(\frac{t+5}{10}\right) S_{L P F, i}(t)\right]\right)\right. \\
& \times \mathbb{F}\left(\left[\Pi\left(\frac{t}{10}\right) S_{L P F, i}(t)\right]\right) \\
& \left.\times \mathbb{F}\left(\left[\Pi\left(\frac{t-5}{10}\right) S_{L P F, i}(t)\right]\right)\right\}^{1 / 3}
\end{aligned}
$$

where $\mathbb{F}$ and $\Pi$ denote the Fourier transform operator and the rectangle function, respectively. In this approach, the length of rectangle function determines the autocorrelation resolution of the detected signals while the overlap length of two adjacent rectangle functions affects the autocorrelation contrast between the vital sign and random body motion signals. Generally speaking, it requires a longer measurement time to achieve a higher autocorrelation resolution by increasing the length of rectangle functions. Moreover, a smaller overlap ratio between two adjacent rectangle functions makes the autocorrelation contrast better to help reduce the effect of random body motion on the detection of vital signs using (20). However, a smaller overlap ratio leads to a lower signal autocorrelation, which makes the extraction of vital signs more difficult. Therefore, process times of 10 and $5 \mathrm{~s}$ are respectively set for the length of rectangle function and the overlap length of two adjacent rectangle functions according to the experimental experience. Fig. 11(b) presents the 


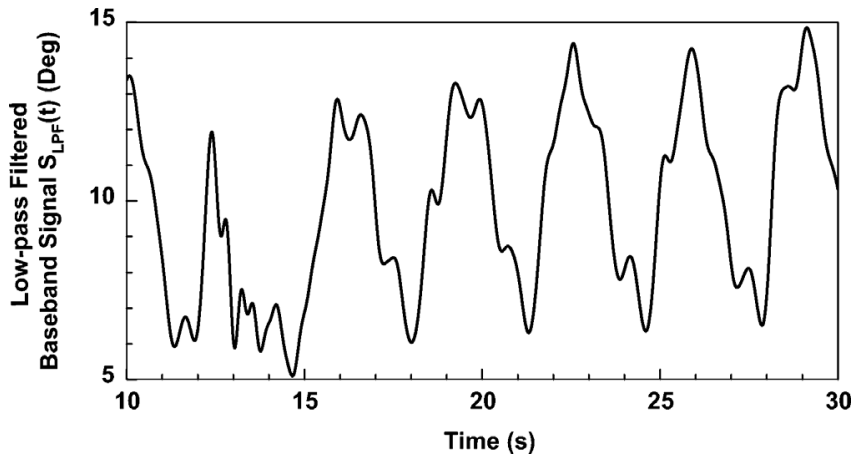

Fig. 12. Low-pass filtered baseband signal for extracting subject 2's vital sign information

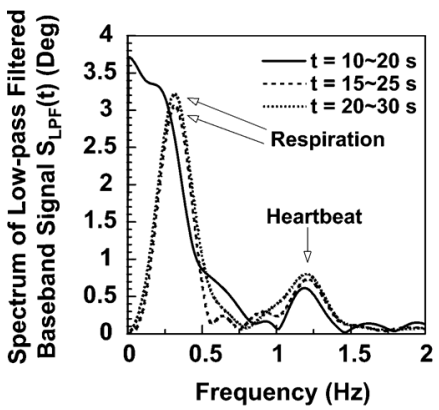

(a)

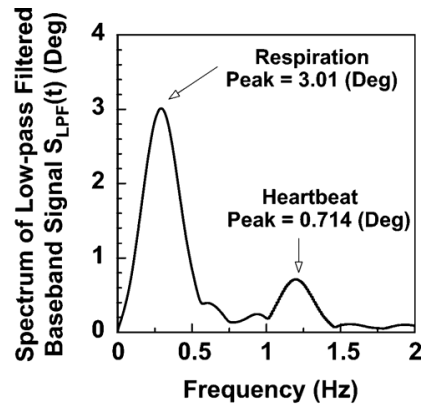

(b)
Fig. 13. Spectra of low-pass filtered baseband signal. (a) Original spectra from the Fourier transform of the waveform of Fig. 12 at different 10-s intervals (b) Improved spectrum using (20).

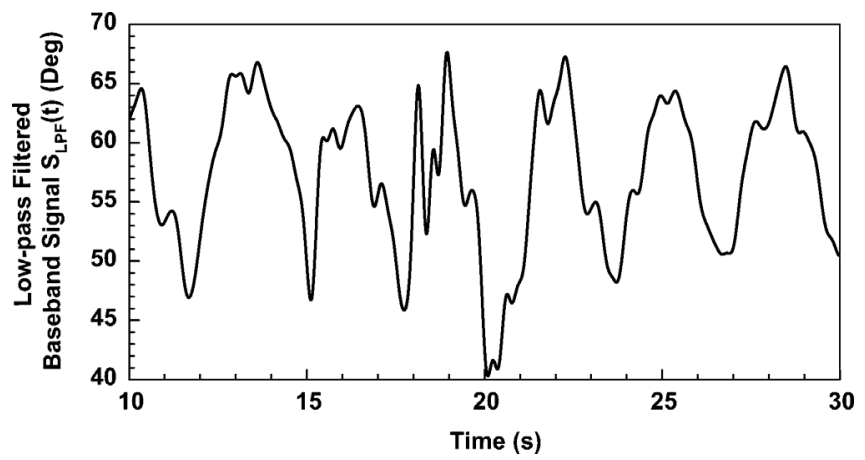

Fig. 14. Low-pass filtered baseband signal for extracting subject 3's vital sign information.

improved spectrum. The vital signs are clearly a breathing rate of $18 \mathrm{breaths} / \mathrm{min}$ and a heart rate of 74 beats $/ \mathrm{min}$.

Fig. 12 plots the signal waveform for subject 2: it includes an irregular fluctuation associated with random body motion from 11 to $15 \mathrm{~s}$. As shown in Fig. 13(a), the resulting spectrum in the period range of $10-20 \mathrm{~s}$ conceals the respiration signal. However, the improved spectrum shows a breathing rate of 17 breaths/min and a heart rate of 72 beats $/ \mathrm{min}$; Also, according to Figs. 14 and 15, subject 3, exhibiting random body motions between 18 and $21 \mathrm{~s}$, has a breathing rate of 22 breaths/min and a heart rate of 71 beats/min. Table II summarizes the sensed vital signs of the subjects. This system can distinguish vital sign signals from random body motion signals with the help of the proposed spectral product approach.

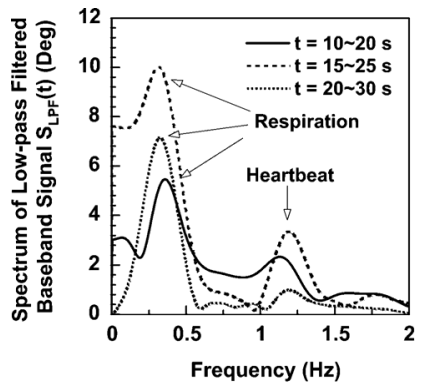

(a)

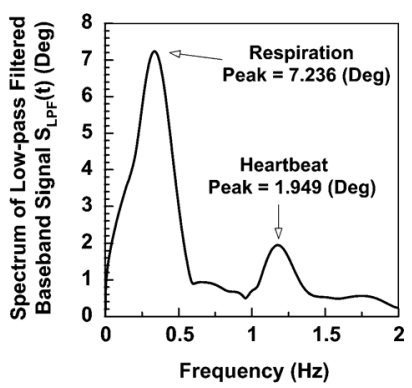

(b)
Fig. 15. Spectra of low-pass filtered baseband signal (a) Original spectra from the Fourier transform of the waveform of Fig. 14 at different 10-s intervals (b) Improved spectrum using (20)

TABLE II

EXTRACTED VITAL Sign INFORMATION FOR DiFFERENT SUBJECTS

\begin{tabular}{cccc}
\hline \hline & Subject 1 & Subject 2 & Subject 3 \\
\hline $\begin{array}{c}\text { Breathing Rate } \\
\text { (breaths/min) } \\
\begin{array}{c}\text { Heart Rate } \\
\text { (beats/min) }\end{array}\end{array}$ & 18 & 17 & 22 \\
\hline \hline
\end{tabular}
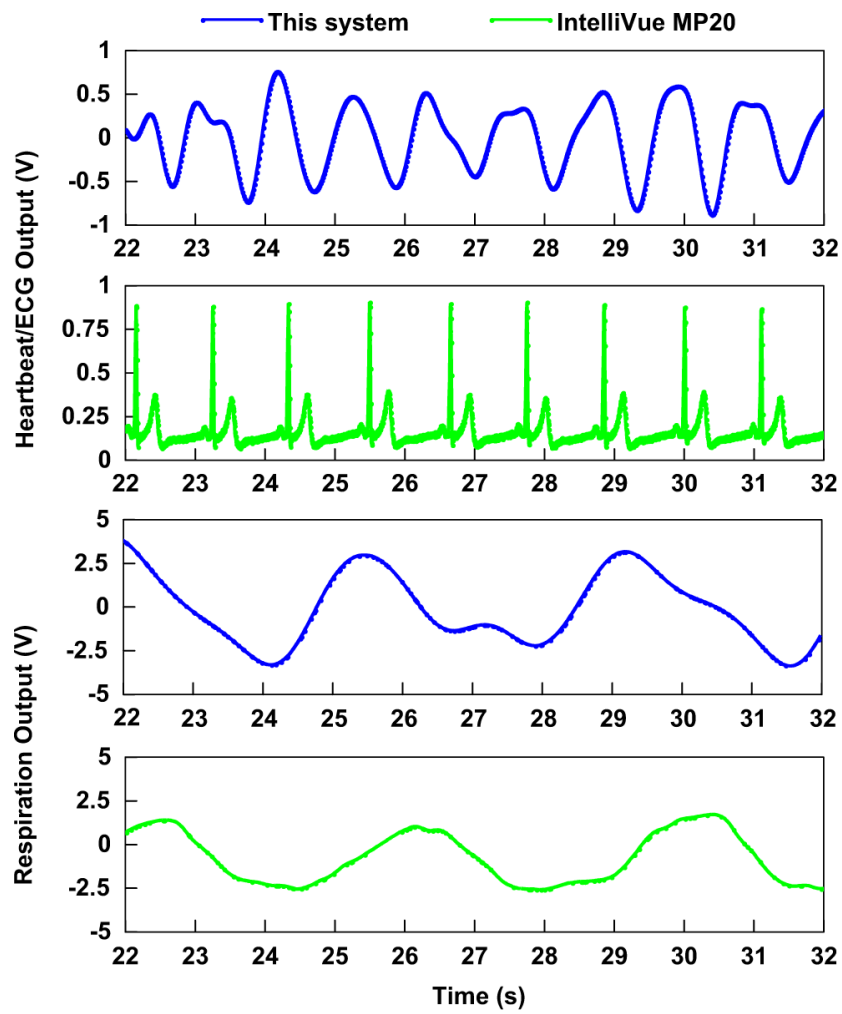

Fig. 16. Comparison of the vital sign measurements from the proposed system with those from IntelliVue MP20.

Fig. 16 compares the measurement results between this system and the commercial patient monitor IntelliVue MP20, where the former is based on the SIL radar method and the latter measures electrocardiogram (ECG) and breathing rates via a three-electrode lead. Both measurements are taken simultaneously and their output waveforms are shown in Fig. 16. With the help of fast Fourier transform, the output waveforms 
TABLE III

COMPARISON OF RECENT INDOOR POSITIONING SySTEMS.

\begin{tabular}{|c|c|c|c|c|c|c|c|c|}
\hline Reference & [4] & [5] & {$[8]$} & [10] & [11] & [18] & [20] & This work \\
\hline $\begin{array}{l}\text { Positioning } \\
\text { algorithm }\end{array}$ & RSS & RSS & TOA, AOA & TDOA & TDOA & RTOF & $\mathrm{AOA}$ & TDOA \\
\hline Technology & $\begin{array}{l}\text { Kernel-based } \\
\text { algorithm }\end{array}$ & Kalman filter & UWB signal & $\begin{array}{l}\text { 1. UWB signal } \\
\text { 2. Peak detection }\end{array}$ & $\begin{array}{l}\text { 1. SIMO TX } \\
\text { 2. E-TDOA }\end{array}$ & $\begin{array}{l}\text { 1. Switched ILO } \\
\text { 2. FMCW radar }\end{array}$ & $\begin{array}{l}\text { Doppler } \\
\text { radar }\end{array}$ & $\begin{array}{l}\text { 1. SIL tag } \\
\text { 2. IL I/Q } \\
\text { receiver }\end{array}$ \\
\hline $\begin{array}{c}\text { Transmit } \\
\text { power }\end{array}$ & N/A & $30 \mathrm{dBm}$ & $\begin{array}{l}23 \mathrm{dBm} \text { in } \\
\text { EIRP }\end{array}$ & $\begin{array}{l}-16 \mathrm{dBm} \text { carrier } \\
\text { leakage }\end{array}$ & $10 \mathrm{dBm}$ & $5 \mathrm{dBm}$ & $-1.24 \mathrm{dBm}$ & $5 \mathrm{dBm}$ \\
\hline $\begin{array}{l}\text { Operating } \\
\text { frequency }\end{array}$ & $2.4 \mathrm{GHz}$ & $0.8665 \mathrm{GHz}$ & $3.4 \mathrm{GHz}$ & $8 \mathrm{GHz}$ & $58 \mathrm{GHz}$ & $34.45 \mathrm{GHz}$ & $2.28 \mathrm{GHz}$ & $2.4 \mathrm{GHz}$ \\
\hline $\begin{array}{l}\text { Bandwidth } \\
\text { per tag }\end{array}$ & $\begin{array}{l}22 \mathrm{MHz} \\
(0.92 \%)\end{array}$ & $\begin{array}{c}3 \mathrm{MHz} \\
(0.35 \%)\end{array}$ & $\begin{array}{c}1400 \mathrm{MHz} \\
(41 \%)\end{array}$ & $\begin{array}{l}6000 \mathrm{MHz} \\
(75 \%)\end{array}$ & $\begin{array}{c}500 \mathrm{MHz} \\
(0.86 \%)\end{array}$ & $500 \mathrm{MHz}(1.5 \%)$ & N/A & $\begin{array}{c}7 \mathrm{MHz} \\
(0.29 \%)\end{array}$ \\
\hline $\begin{array}{l}\text { Sensing } \\
\text { Range }\end{array}$ & $36 \times 42 \mathrm{~m}^{2}$ & $6 \times 5.5 \mathrm{~m}^{2}$ & $20 \times 20 \mathrm{~m}^{2}$ & N/A & $0.2 \times 0.2 \mathrm{~m}^{2}$ & $\begin{array}{c}11.5 \mathrm{~m} \\
\text { (Distance) }\end{array}$ & $6 \times 7 \mathrm{~m}^{2}$ & $8 \times 8 \mathrm{~m}^{2}$ \\
\hline $\begin{array}{l}\text { Number of } \\
\text { receivers }\end{array}$ & 33 & 8 & 4 & 6 & 4 & 1 & 2 & 3 \\
\hline $\begin{array}{c}\text { Number of } \\
\text { tags }\end{array}$ & 1 & 2 & 1 & 1 & 4 & 1 & 3 & 3 \\
\hline Accuracy & $231 \mathrm{~cm}$ & $10 \mathrm{~cm}$ & $25 \mathrm{~cm}$ & $0.245 \mathrm{~cm}$ & $2 \mathrm{~cm}$ & $10 \mathrm{~cm}$ & $30 \mathrm{~cm}$ & $10 \mathrm{~cm}$ \\
\hline
\end{tabular}

from two different systems yield consistent estimates of 55 and 17 times/min for heart and breathing rate, respectively.

Table III compares recently developed indoor positioning systems. The RSS systems, developed by [4] and [5], involve more receivers and less bandwidth than other systems. To improve positioning accuracy, [4] and [5] develop a kernelized distance calculation algorithm and a positioning Kalman filter, respectively, to process the measured signal strengths from various receiving units. [8] combines TOA and AOA positioning algorithms with a $1400 \mathrm{MHz}$ channel bandwidth to mitigate the positioning inaccuracy in a dense cluttered environment. The other AOA system [20] utilizes Doppler radars to detect a subject from his or her respiration signals. It therefore provides a tag-free wireless positioning system for human subjects. The switched-beam phased antenna arrays of [8] and [20] involve four antenna elements to position the desired target, and the azimuth resolution is further improved in each case by increasing the number of antenna elements. [18] uses a millimeter-wave FMCW radar as a measuring unit and a switched injection-locked oscillator as a backscatter transponder tag for RTOF estimation. This transponder architecture significantly amplifies the response signals and thus enlarges the sensing range. The positioning accuracy reaches $10 \mathrm{~cm}$. In the prior art using TDOA method, [10] utilizes a 300 ps Gaussian pulse to modulate an $8 \mathrm{GHz}$ carrier signal. Additionally, many schemes are applied to synchronize receiving units on the picosecond scale so as to achieve a best accuracy of $0.245 \mathrm{~cm}$. [11] proposes a single input and multiple output (SIMO) approach with an enhanced TDOA (E-TDOA) algorithm to mitigate the multipath problem. It provides accurate positioning in a small environment. Generally, all previous methods involve a trade-off among accuracy, channel bandwidth, and system complexity. In contrast, this work develops SIL tags and IL $\mathrm{I} / \mathrm{Q}$ receivers to implement a TDOA-based indoor positioning system without using RF reference signals. It achieves a $10-\mathrm{cm}$ accuracy with a 7-MHz channel bandwidth. In comparison, the proposed system has the smallest fractional bandwidth per tag and a relatively simple architecture with comparable accuracy. Moreover, with the help of FDMA technique, the system is validated to simultaneously detect information about the vital signs and positions of multiple subjects.

\section{CONCLUSION}

This work presents a sensing system that combines TDOA and SIL radar technology to provide simultaneous information about the vital signs and positions of subjects in an indoor environment. The system architecture consists of mainly SIL tags and IL I/Q receivers to eliminate the reference signal synchronization issue that arises in conventional TDOA methods. The FDMA technique is used to identify multiple individuals in the sensing range. In a cubicle office of $8 \times 8 \mathrm{~m}^{2}$, the $2.4 \mathrm{GHz}$ prototype receivers locate three subjects with an error of under $10 \mathrm{~cm}$. Moreover, the proposed spectral product approach provides accurate and reliable information of the cardiopulmonary activity of each subject who is standing with fidgeting movements.

\section{ACKNOWLEDGMENT}

The authors wish to thank the Advanced Micro Sensor Laboratory, ITRI Center for Measurement Standards, Hsinchu, Taiwan, for providing the IntelliVue MP20 patient monitor for this research. Their gratitude is also expressed to Mr. Y.-C. Chiu and Mr. Y.-R. Chou who volunteered to serve in the sensing experiments. 


\section{REFERENCES}

[1] H. Liu, H. Darabi, P. Banerjee, and J. Liu, "Survey of wireless indoor positioning techniques and systems," IEEE Trans. Syst. Man Cybern. C., vol. 37, no. 6, pp. 1067-1080, Nov. 2007.

[2] R. Miesen, R. Ebelt, F. Kirsch, T. Schafer, G. Li, H. Wang, and M. Vossiek, "Where is the tag?," IEEE Microw. Mag., vol. 12, no. 7, pp. S49-S63, Dec. 2011.

[3] X. Li, "Collaborative localization with received-signal strength in wireless sensor networks," IEEE Trans. Veh. Technol., vol. 56, no. 6, pp. 3807-3817, Nov. 2007.

[4] A. Kushki, K. N. Plataniotis, and A. N. Venetsanopoulos, "Kernelbased positioning in wireless local area networks," IEEE Trans. Mobile Comput., vol. 6, no. 6, pp. 689-705, Jun. 2007.

[5] S. S. Saab and Z. S. Nakad, "A standalone RFID indoor positioning system using passive tags," IEEE Trans. Ind. Electron., vol. 58, no. 5, pp. 1961-1970, May 2011.

[6] B. Dennis, J.-B. Pierrot, and C. Abou-Rjeily, "Joint distributed synchronization and positioning in UWB ad hoc networls using TOA," IEEE Trans. Microw. Theory Tech., vol. 54, no. 4, pp. 1896-1911, Apr. 2006.

[7] S. Gezici, Z. Tian, G. B. Giannakls, H. Kobayashi, A. F. Molisch, H. V. Poor, and Z. Sahinoglu, "Localization via ultra-wideband radios: a look at positioning aspects of future sensor networks," IEEE Signal Process. Mag., vol. 22, no. 4, pp. 70-84, Jul. 2005.

[8] Y. Luo and C. L. Law, "Indoor positioning using UWB-IR signals in the presence of the dense multipath with path overlapping," IEEE Trans. Wireless Commun., vol. 11, no. 10, pp. 3734-3743, Oct. 2012.

[9] W. A. Gardner and C.-K. Chen, "Signal-selective time-difference-of-arrival estimation for passive location of man-made signal sources in highly corruptive environments, part I: theory and method," IEEE Trans. Signal Process., vol. 40, no. 5, pp. 1168-1184, May 1992.

[10] M. R. Mahfouz, C. Zhang, B. C. Merkl, M. J. Kuhn, and A. E. Fathy, "Investigation of high-accuracy indoor 3-D positioning using UWB technology," IEEE Trans. Microw. Theory Tech., vol. 56, no. 6, pp. 1316-1330, Jun. 2008

[11] M. Bocquet, C. Loyez, and A. Benlarbi-Delai, "Using enhanced-TDOA measurement for indoor positioning," IEEE Microw. Wireless Compon. Lett., vol. 15, no. 10, pp. 612-614, Oct. 2005

[12] M. Vossiek, L. Wiebking, P. Gulden, J. Wieghardt, C. Hoffmann, and P. Heide, "Wireless local positioning," IEEE Microw. Mag., vol. 4, no. 4, pp. 77-86, Dec. 2003.

[13] J. R. Riley, A. D. Smith, D. R. Reynolds, A. S. Edwards, J. L. Osborne, I. H. Williams, N. L. Carreck, and G. M. Poppy, "Tracking bees with harmonic radar," Nature, vol. 379, pp. 29-30, Jan. 1996.

[14] N. Tahir and D. G. Brooker, "Recent developments and recommendations for improving harmonic radar tracking systems," in Proc. 5th Eur. Antenna Propagation Conf., Rome, Italy, Apr. 2011, pp. 1531-1535.

[15] B. G. Colpitts and G. Boiteau, "Harmonic radar transceiver design: minituare tags for insect tracking," IEEE Trans. Antennas Propag., vol. 52, no. 11, pp. 2825-2832, Nov. 2004.

[16] Z.-M. Tsai, P.-H. Jau, N.-C. Kuo, J.-C. Kao, K.-Y. Lin, F.-R. Chang, and E.-C. Yang, "A high-range-accuracy and high-sensitivity harmonic radar using pulse pseudorandom code for bee searching," IEEE Trans. Microw. Theory Tech., vol. 61, no. 1, pp. 666-675, Jan. 2013.

[17] M. Vossiek and P. Gulden, "The switched injection-locked oscillator: a novel versatile concept for wireless transponder and localization systems," IEEE Trans. Microw. Theory Tech., vol. 56, no. 4, pp. 859-866, Apr. 2008.

[18] A. Strobel, C. Carlowitz, R. Wolf, F. Ellinger, and M. Vossiek, "A millimeer-wave low-power active backscatter tag for FMCW radar system," IEEE Trans. Microw. Theory Tech., vol. 61, no. 5, pp. 1964-1972, May 2013.

[19] R. Peng and M. L. Sichitiu, "Angle of arrival localization for wireless sensor networks," in Proc. IEEE Commun. Soc. Conf. Sensor Ad Hoc Commun. Networks., Reston, VA, USA, Sep. 2006, pp. 374-382.

[20] Y.-S. Su, C.-C. Chang, J.-J. Guo, and S.-F. Chang, "2-D wireless human subjects positioning system based on respiration detections," in Proc. IEEE MTT-S Int. Microw. Symp. Dig., Montreal, QC, Canada, Jun. 2012, pp. 1-3.

[21] C. Li, V. M. Lubecke, O. Boric-Lubecke, and J. Lin, "A review on recent advances in Doppler radar sensors for noncontact healthcare monitoring," IEEE Trans. Microw. Theory Tech., vol. 61, no. 5, pp. 2046-2060, May 2013.
[22] G. Wang, C. Gu, T. Inoue, and C. Li, "Hybrid FMCW-interferometry radar system in the $5.8 \mathrm{GHz}$ ISM band for indoor precise position and motion detection," in Proc. IEEE MTT-S Int. Microwave Symp. Dig., Seattle, WA, USA, Jun. 2013, pp. 1-4.

[23] M. Mercuri, P. J. Soh, G. Pandey, P. Karsmakers, G. A. E. Vandenbosch, P. Leroux, and D. Schreurs, "Analysis of an indoor biomedical radar-based system for health moniroting," IEEE Trans. Microw. Theory Tech., vol. 61, no. 5, pp. 2061-2068, May 2013.

[24] F.-K. Wang, C.-J. Li, C.-H. Hsiao, T.-S. Horng, J. Lin, K.-C. Peng, J.-K. Jau, J.-Y. Li, and C.-C. Chen, "A novel vital-sign sensor based on a self-injection-locked oscillator," IEEE Trans. Microw. Theory Tech., vol. 58 , no. 12 , pp. $4112-4120$, Dec. 2010

[25] F.-K. Wang, T.-S. Horng, K.-C. Peng, J.-K. Jau, Li J.-Y, and C.-C. Chen, "Single-antenna Doppler radars using self and mutual injection locking for vital sign detection with random body movement cancellation," IEEE Trans. Microw. Theory Tech., vol. 59, no. 12, pp. $3577-3587$, Dec. 2011

[26] F.-K. Wang, T.-S. Horng, K.-C. Peng, J.-K. Jau, Li J.-Y, and C.-C. Chen, "Detection of concealed individuals based on their vital signs by using a see-through-wall imaging system with a self-injection-locked radar," IEEE Trans. Microw. Theory Tech., vol. 61, no. 1, pp. 696-704 Jan. 2013

[27] F.-K. Wang, T.-S. Horng, K.-C. Peng, Li J.-Y, and C.-C. Chen, "Combining SIL tag and IL receiver for concurrent vital sign and position sensing," in Proc. IEEE MTT-S Int. Microwave Symp. Dig, Seattle, WA, Jun. 2013, pp. 1-4.

[28] R. Adler, "A study of locking phenomena in oscillators," Proc. IRE, vol. 34, no. 6, pp. 351-357, Jun. 1946.

[29] T. Yilmaz, R. Foster, and Y. Hao, "Detecting vital signs with wearable wireless sensors," Sensors, vol. 10, pp. 10837-10862, Dec. 2010.

[30] C.-T. Chen, C.-H. Hsiao, T.-S. Horng, K.-C. Peng, and C.-J. Li, "Cognitive polar receiver using two injection-locked oscillator stages," IEEE Trans. Microw. Theory Tech., vol. 59, no. 12, pp. 3484-3493, Dec. 2011.

[31] B.-K. Park, O. Boric-Lubecke, and V. M. Lubecke, “Arctangent demodulation with DC offset compensation in quadrature Doppler radar receiver systems," IEEE Trans. Microw. Theory Tech., vol. 55, no. 5, pp. 1073-1079, May 2007.

[32] Y. Qi, H. Kobayashi, and H. Suda, "Analysis of wireless geolocation in a non-line-of-sight environment," IEEE Trans. Wireless Commun., vol. 5, no. 3, pp. 672-681, Mar. 2006.

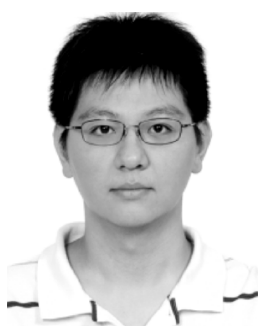

Fu-Kang Wang (S'10-M'13) was born May 15, 1985 , in Kaohsiung, Taiwan. He received the B.S.E.E., M.S.E.E. and Ph.D. degrees from National Sun Yat-Sen University, Kaohsiung, Taiwan, in 2007, 2009, and 2013, respectively.

He is currently a Postdoctoral Research Fellow in electrical engineering at National Sun Yat-Sen University. His research interest is focused on RF sensing techniques.

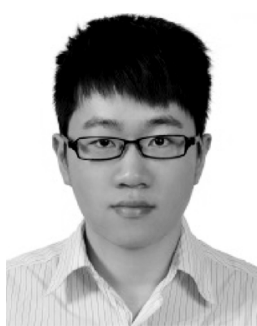

Chia-Hui Fang was born in Tainan, Taiwan, on July 19, 1989. He received the B.S.E.E degree from National University of Kaohsiung, Kaohsiung, Taiwan, in 2011, and the M.S.E.E. degree from National Sun Yat-Sen University, Kaohsiung, Taiwan, in 2013.

His research work is dedicated to the development of wireless sensor network for biomedical applications. 


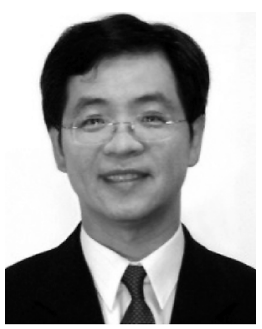

Tzyy-Sheng Horng (S'88-M'92-SM'05) was born in Taichung, Taiwan, on December 7, 1963. $\mathrm{He}$ received the B.S.E.E. degree from National Taiwan University, Taipei, Taiwan, in 1985, and the M.S.E.E. and Ph.D. degrees from the University of California at Los Angeles (UCLA), Los Angeles, CA, USA, in 1990 and 1992, respectively.

Since August 1992, he has been with the Department of Electrical Engineering, National Sun Yat-Sen University, Kaohsiung, Taiwan, where he was the Director of the Telecommunication Research and Development Center (2003-2008), Director of the Institute of Communications Engineering (2004-2007), and where he is currently a Distinguished Professor. He has authored or coauthored over 200 technical publications published in refereed journals and conferences proceedings, mostly in IEEE publications. $\mathrm{He}$ holds over ten patents. His research interests include RF and microwave integrated circuits (ICs) and components, RF signal integrity for wireless system-in package, digitally assisted RF technologies, and green radios for cognitive sensors and Doppler radars.

Dr. Horng has served on several Technical Program Committees of international conferences including the International Association of Science and Technology for Development (IASTED) International Conference on Wireless and Optical Communications, the IEEE Region 10 International Technical Conference, the IEEE International Workshop on Electrical Design of Advanced Packaging and Systems (EDAPS), the Asia-Pacific Microwave Conference (APMC), the IEEE Radio and Wireless Symposium (RWS), and the Electronic Components and Technology Conference (ECTC). He has also served on the Project Review Board of the Programs of Communications Engineering and Microelectronics Engineering, National Science Council, Taiwan, and the Technology Development Program of the Ministry of Economic Affairs, Taiwan. He is the founder chair of the IEEE Microwave Theory and Techniques Society (IEEE MTT-S) Tainan Chapter. He is currently an associate editor for the IEEE TRANSACTIONS ON MICROWAVE THEORY AND TECHNIQUES. $\mathrm{He}$ is a member of the IEEE MTT-S Technical Committee MTT-10 and MTT-20. He was the recipient of the 1996 Young Scientist Award presented by the International Union of Radio Science, the 1998 Industry-Education Cooperation Award presented by the Ministry of Education, Taiwan, and the 2010 Distinguished Electrical Engineer Award presented by the Chinese Institute of Electrical Engineering, Kaohsiung Branch, Taiwan. He was also the recipient of the 2011 Advanced Semiconductor Engineering (ASE) Inc. Chair Professorship and the 2012 Outstanding Research Award of National Sun Yat-Sen University.

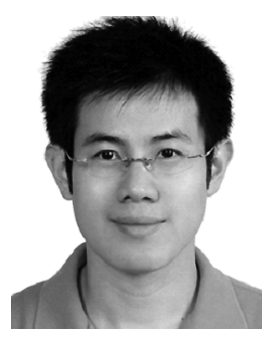

Kang-Chun Peng (S'00-M'05) was born February 18, 1976, in Taipei, Taiwan. He received the B.S.E.E., M.S.E.E., and Ph.D. degrees from National Sun Yat-Sen University, Kaohsiung, Taiwan, in 1998, 2000, and 2005, respectively.

$\mathrm{He}$ is currently an Assistant Professor with the Department of Computer and Communication Engineering, National Kaohsiung First University of Science and Technology, Kaohsiung, Taiwan. His current research interests are in the area of delta-sigma modulation techniques, low noise PLLs, low-power VCOs and modulated frequency synthesizers.

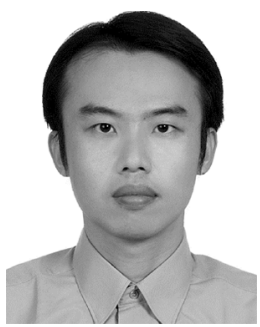

Jian-Yu Li (S'07-M'13) was born in Pingtung, Taiwan. He received the B.S.E.E and M.S.E.E degrees from National Sun Yat-Sen University, Kaohsiung, Taiwan, in 1998 and 2000, respectively, and the $\mathrm{Ph} . \mathrm{D}$. degree in communication engineering from National Chiao Tung University, Hsinchu, Taiwan, in 2013.

In 2000, he joined the Information and Communications Research Laboratories, Industrial Technology and Research Institute (ITRI), Hsinchu, Taiwan. His research interests are in the area of microwave integrated circuits and passive components design.

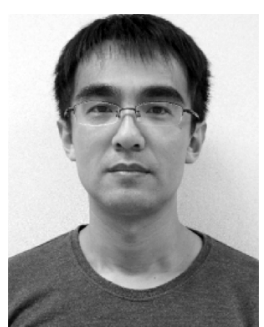

Cheng-Chung Chen was born in Hsinchu, Taiwan, in 1975. He received the B.S. degree in electrical engineering from National Sun Yet-Sen University, Kaoshiung, Taiwan in 1997, and the M.S. and Ph.D. degrees in communication engineering from National Chiao-Tung University, Hsinchu, Taiwan, in 1999 and 2003, respectively.

In 2003, He joined the Information and Communications Research Laboratories, Industrial Technology and Research Institute (ITRI), Hsinchu, Taiwan. His research interests include RF front-end 